\title{
Using Imagination to Understand the Neural Basis of Episodic Memory
}

\author{
Demis Hassabis, Dharshan Kumaran, and Eleanor A. Maguire \\ Wellcome Trust Centre for Neuroimaging, Institute of Neurology, University College London, London WC1N 3BG, United Kingdom
}

\begin{abstract}
Functional MRI (fMRI) studies investigating the neural basis of episodic memory recall, and the related task of thinking about plausible personal future events, have revealed a consistent network of associated brain regions. Surprisingly little, however, is understood about the contributions individual brain areas make to the overall recollective experience. To examine this, we used a novel fMRI paradigm in which subjects had to imagine fictitious experiences. In contrast to future thinking, this results in experiences that are not explicitly temporal in nature or as reliant on self-processing. By using previously imagined fictitious experiences as a comparison for episodic memories, we identified the neural basis of a key process engaged in common, namely scene construction, involving the generation, maintenance and visualization of complex spatial contexts. This was associated with activations in a distributed network, including hippocampus, parahippocampal gyrus, and retrosplenial cortex. Importantly, we disambiguated these common effects from episodic memory-specific responses in anterior medial prefrontal cortex, posterior cingulate cortex and precuneus. These latter regions may support self-schema and familiarity processes, and contribute to the brain's ability to distinguish real from imaginary memories. We conclude that scene construction constitutes a common process underlying episodic memory and imagination of fictitious experiences, and suggest it may partially account for the similar brain networks implicated in navigation, episodic future thinking, and the default mode. We suggest that additional brain regions are co-opted into this core network in a task-specific manner to support functions such as episodic memory that may have additional requirements.
\end{abstract}

Key words: fMRI; episodic memory; imagination; scene; construction; recollection

\section{Introduction}

A rich recollective experience is a defining characteristic of episodic memory recall, the memory for our everyday personal experiences (Tulving, 2002). Recollection of this type of memory is widely accepted to be a reconstructive process (Bartlett, 1932; Schacter et al., 1998; Conway and Pleydell-Pearce, 2000; Rubin et al., 2003; Hassabis and Maguire, 2007; Schacter and Addis, 2007) as opposed to the simple retrieval of a perfect holistic record. Thus episodic memory recall can be conceptually divided into a number of component processes including a sense of subjective time (Tulving, 2002), self-processing (Conway and PleydellPearce, 2000), visual imagery (Rubin et al., 2003), narrative structure (Rubin et al., 2003), retrieval of semantic information (Wheeler et al., 1997) and multimodal details (Wheeler et al., 2000; Gottfried et al., 2004), and feelings of familiarity (Wagner et al., 2005). Although numerous fMRI studies investigating the neural basis of episodic memory recall (Maguire, 2001; Svoboda et al., 2006; Cabeza and St Jacques, 2007), and more recently the closely related task of future thinking (Atance and O'Neill, 2001;

Received June 25, 2007; revised Nov. 12, 2007; accepted Nov. 14, 2007.

This work was supported by the Brain Research Trust and the Wellcome Trust. We thank Peter Aston for technical assistance.

The authors declare no competing financial interests.

Correspondence should be addressed to Demis Hassabis, Wellcome Trust Centre for Neuroimaging, Institute of Neurology, University College London, 12 Queen Square, London WC1N 3BG, UK. E-mail: d.hassabis@fil.ion.ucl.ac.uk.

DOI:10.1523/JNEUROSCI.4549-07.2007

Copyright $\odot 2007$ Society for Neuroscience $\quad 0270-6474 / 07 / 2714365-10 \$ 15.00 / 0$
Addis et al., 2007; Szpunar et al., 2007), have revealed a consistent and distributed network of associated brain regions, surprisingly little is understood about the contributions individual areas make to the overall recollective experience.

To progress this issue further we used a novel paradigm in which subjects, as well as recollecting past memories, had to imagine new fictitious experiences (Hassabis et al., 2007) during fMRI scanning. Episodic memory and imagining fictitious experiences share striking similarities in terms of the psychological processes engaged (Conway and Pleydell-Pearce, 2000; Greenberg and Rubin, 2003; Schacter and Addis, 2007) including imagery (Rubin et al., 2003) and the retrieval of relevant semantic information (Wheeler et al., 1997). Moreover, both tasks involve the process of "scene construction" (Hassabis and Maguire, 2007; Hassabis et al., 2007), that is, the generation, maintenance and visualization of a complex spatial setting in which an event (real or imaginary) can be mentally experienced (Burgess et al., 2001; Hassabis and Maguire, 2007). Note that this kind of complex "scene" imagery differs markedly from "simple" imagery (Kosslyn et al., 2001) (e.g., for faces or single objects) in that it requires the binding of disparate (possibly multimodal) elements of a scene into a coherent whole. As such, scene construction likely recruits a set of additional processes underpinned by regions such as the hippocampus (Hassabis et al., 2007) not thought to be involved in simple imagery (Rosenbaum et al., 2004). Critically, imagining fictitious scenarios, in contrast to tasks that require thinking about plausible personal future events such as those 
used in several recent episodic memory studies (Addis et al., 2007; Szpunar et al., 2007), results in experiences that are not explicitly temporal in nature. Furthermore, a purely created imagined experience does not have the same reliance or effect on the imaginer's self-schema compared with a real episodic memory (Conway and Pleydell-Pearce, 2000; Gallagher, 2000) although both likely involve the adoption of an egocentric viewpoint (Burgess, 2006) on the part of the imaginer. By using imagining fictitious experiences as a comparison task for episodic memory, it is possible to address some key questions not amenable to previous studies (Addis et al., 2007; Szpunar et al., 2007).

Specifically, in the present study we asked subjects to recall very recent episodic memories, retrieve fictitious experiences previously constructed in a prescan interview 1 week before, and to construct new fictitious experiences during fMRI scanning. We reasoned that brain regions engaged in common during episodic memory retrieval and imagined experiences would likely support scene construction regardless of whether experiences were real or fictitious (Hassabis et al., 2007). We further hypothesized that brain areas selectively active during episodic memory recall would mediate specific aspects of remembering one's own past experiences including those related to self-schema (Conway and Pleydell-Pearce, 2000; Gallagher, 2000) and mental time travel (Tulving, 2002). In this way, we hoped not only to characterize the neural circuitry underlying the (re)construction of complex scenes, but also to gain new insights into the component processes supported by different brain regions in the episodic memory network. As such, we set out to functionally deconstruct a cortical network that has recently been suggested (Buckner and Carroll, 2007; Hassabis and Maguire, 2007) to support not just episodic memory, but also numerous other high-level cognitive functions including future thinking (Addis et al., 2007; Szpunar et al., 2007), spatial navigation (Burgess et al., 2002; Hartley et al., 2003), theory of mind (Frith and Frith, 2003), the default network (Raichle et al., 2001), and perhaps even daydreaming (Mason et al., 2007).

\section{Materials and Methods}

Subjects. Twenty-one healthy, right-handed, native English speakers participated in the experiment ( 10 males; mean \pm SD age, $24.8 \pm 3.8$ years; age range, $18-31$ years). All subjects gave informed written consent to participation in accordance with the local research ethics committee.

Prescan interview. Interview sessions were conducted with subjects $\sim 1$ week before scanning (mean \pm SD, $6.24 \pm 1.45 \mathrm{~d}$ ). Subjects sat facing the experimenter and engaged in four tasks (for conditions summary, see Table 1). They were told at the outset to try and remember as much as possible about the things they were going to do during the interview session as their memory would be tested in the subsequent scanning session. First they were shown 10 pictures of everyday objects on a computer screen (e.g., a red fire extinguisher) [condition: real objects (RO)]. Each object was shown centrally in isolation for $20 \mathrm{~s}$ set against a plain white background along with an accompanying line of text (in black along the bottom of the screen) describing its key features (e.g., "a red fire extinguisher with a silver label and black nozzle"). Subjects were instructed to use the time to try and remember as many details about the object as possible and also to form a strong mental image of the object. Then short descriptions of 10 additional everyday objects (e.g., "a fancy gold-plated pen with a silver nib and the initials JT engraved along the casing") were read out by the experimenter [condition: imagined objects (IO)]. Subjects closed their eyes and were given $20 \mathrm{~s}$ to form a vivid and detailed mental picture of the object. The importance of visualizing the object either against a plain background or simply floating in mid-air, i.e., in the absence of any background context, was stressed and this was verified by the experimenter after each object was imagined.

Next, subjects were asked to describe 10 fictitious scenes in response to short verbal cues read out by the experimenter [condition: imagined scene (IS)] outlining a range of commonplace scenarios (e.g., "Imagine you are lying on a sandy beach in a tropical bay. Describe what you can see, hear, smell, and feel in as much detail as possible.") (Hassabis et al., 2007). Commonplace, ordinary settings were chosen to minimize the difficulty level and to be as independent from a subject's innate creative ability as possible. The scenarios also purposely encompassed a wide variety of different subject matters from the manmade to the natural and the busy to the isolated to ensure there were no content biases. Thus the imagined scenarios could be considered as "episodic-like" experiences in that they involved the salient experiencing of complex scenes that included people and actions, with the implicit constraint that any event imagined to be taking place necessarily had a relatively short duration and occurred in one context. Subjects were asked to close their eyes and vividly imagine the scene and then given $2-3$ min to describe it in as much (multimodal) detail as possible. Importantly subjects were explicitly told not to recount an actual memory or any part of one or something they planned to do but rather to create something completely new. After each scenario, participants were asked to rate their imagined scenes for vividness (1, not vivid. . .5, very vivid) and for similarity to a real memory ( 1 , nothing like any memory. . . 5, exactly like a memory). All descriptions were digitally recorded for later scoring purposes. Finally, 10 recent real episodic memories were elicited from the subjects [condition: real memory (RM)]. Memories had to be emotionally neutral, specific in time and place, and vividly recalled to be accepted as a stimulus. If a memory involved a long sequence of events (e.g., going to the cinema one evening) subjects were instructed to focus on a single shorter temporal element of that event and describe just it and its immediate location (e.g., buying the ticket at the ticket booth in the cinema lobby) so as to qualitatively match the imagined scene conditions as closely as possible [i.e., a snapshot (Hassabis et al., 2007)]. Subjects were encouraged to think of memories that were set in different distinct environments.

Overall it is important to note that because tasks were performed in a single prescan session in one sitting and one context, all conditions were matched for effects related to the memory of the interview itself. In addition, only very recent autobiographical memories (mean $\pm \mathrm{SD}$ age, $12.82 \pm 2.77 \mathrm{~d}$ ) were used as stimuli to be temporally comparable with the imagined memories.

Stimuli. Stimuli for the scanning session consisted of simple one-line text cues presented centrally on the screen in white on a plain black background (Fig. 1). Each cue started with a keyword indicating the trial type followed by a very brief description of the scene or object to be visualized. Four keywords were used: "Recall" indicated that the description that followed was either a real autobiographical memory (different for each subject) or an object that had been seen in the interview session (RM and RO conditions); "Recreate" indicated trials in which an imagined scene or object previously created in the interview session was to be reconstructed (IS and IO conditions); "Imagine" prefixed trials in which a new fictitious scene or object was to be imagined for the first time in the scanner [new scene (NS) and new object (NO) conditions]. "Focus" was 


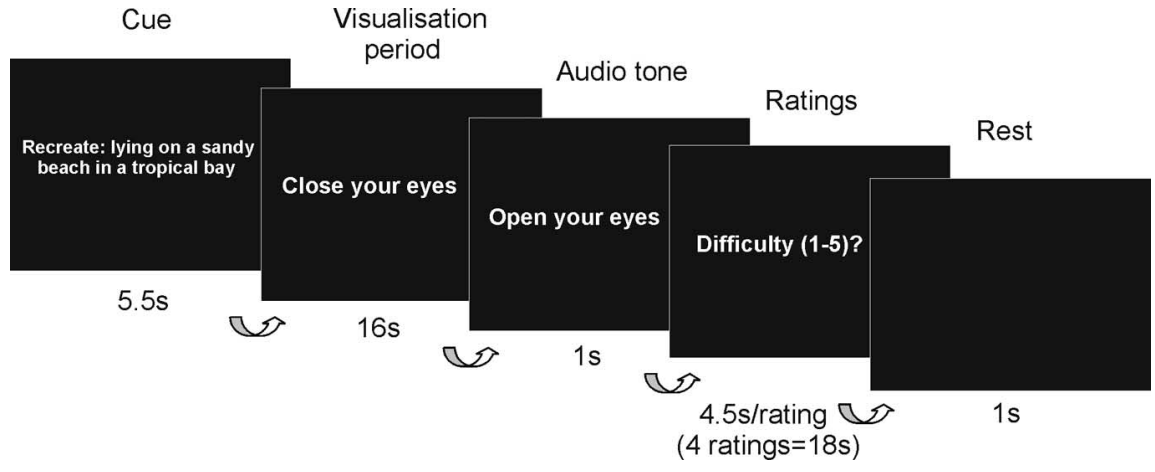

Figure 1. Experimental design. A text cue, prefixed by an instruction keyword denoting trial type ["Recall," "Recreate," "Imagine" (see Materials and Methods)], was presented for $5.5 \mathrm{~s}$ describing the scene or object to be visualized. Subjects were then instructed to close their eyes and begin visualizing the scene or object in as much detail as possible for the entire $16 \mathrm{~s}$ duration. A simple audio tone, played through headphones and lasting $1 \mathrm{~s}$, indicated the end of the visualization period at which point the subject opened their eyes. Subjects then used a five-button MR-compatible box to rate their just visualized scene or object across four ratings on five-point scales: difficulty, vividness, coherence, and memory. Subjects were given $4.5 \mathrm{~s}$ to respond per rating, resulting in an overall rating period of $18 \mathrm{~s}$. This was followed by a $1 \mathrm{~s}$ rest period in which a blank screen was presented before the start of the next trial.

used to indicate a low imagery baseline task where subjects had to imagine and then focus on a white crosshair on a black background.

Task. Outside the scanner, the instructions and keywords were explained to subjects before scanning, and they received extensive training to ensure they were thoroughly familiarized with all aspects of the task. Subjects also had a practice session in the scanner before the main scanning sessions consisting of one trial per condition. There were 7 conditions ( 3 scene conditions: RM, IS, NS; 3 object conditions: RO, IO, NO; and the low imagery baseline condition) with 10 trials per condition, yielding 70 trials in total. As in the prescan interview session, subjects were explicitly told that for the scenes and objects they would newly imagine in the scanner (NS, NO) they should not give an actual memory or any part of one but rather to create something completely new. Scanning consisted of four main sessions lasting $\sim 11$ min each during which 17 or 18 trials were pseudorandomly presented such that 2 or 3 trials were presented per condition, and never the same condition twice in a row. Text cues remained on the screen for $5.5 \mathrm{~s}$ and were then replaced by a "close your eyes" text instruction (Fig. 1). At this point subjects were instructed to close their eyes immediately and begin visualizing the scene or object (the "visualization period") as vividly and, in the case of the "memory" conditions (RM, RO, IS, IO), as accurately as possible. Extensive piloting in the scanning environment revealed that subjects required $\sim 16$ s to comfortably perform the visualization tasks. Subjects were required to focus on the scene or object they were imagining, adding more details if necessary, for the entire $16 \mathrm{~s}$ duration of the visualization period. A simple $1 \mathrm{~s}$ audio tone signaled the end of the visualization period (at which point subjects opened their eyes) and the start of the ratings phase. Using an MR-compatible five-button keypad, subjects scored their justvisualized scene or object across four ratings: difficulty (how hard was the trial: 1 , easy. . . 5, hard), vividness (salience of the imagery: 1 , not vivid. . . 5 , very vivid), coherence (contiguousness of the spatial context: 1 , fragmented. . 5, completely contiguous), and memory (how much like a memory the visualized scene or object was: 1 , nothing like a memory. . . 5 , exactly like a memory). For each rating subjects were given $4.5 \mathrm{~s}$ to respond. This was followed by a $1 \mathrm{~s}$ period of rest before the next cue was presented. The scenes and objects used for the new imagination conditions (NS, NO) and those imagined in the prescan interview (IS, IO) were counter-balanced across subjects.

Debriefing and scoring. After scanning, subjects were thoroughly debriefed. Subjects were first tested on their memory for all the seen (RO) and imagined objects (IO) from the prescan interview. The cues for the objects were read out and subjects provided as many details as they could remember. An object was regarded as accurately remembered if the subject was able to correctly name at least two salient features of the object correctly with no incorrect details. Then two real memories (RM), two previously imagined scenes (IS), and two newly imagined scenes (NS) were randomly selected and subjects were asked to describe in detail what they had visualized in the scanner. These descriptions were digitally recorded for later transcription and scoring. The number of distinct details was calculated, and for the previously imagined scene condition (IS) the details were also compared with those described when that same scene was initially created in the prescan interview, thus providing an objective measure of how accurately the scene had been recreated in the scanner. Subjects were then asked how emotional the three main scene conditions made them feel overall on a scale from -3 (negative) to +3 (positive). Finally, they were also asked to rate on a five-point scale how much they felt themselves to be an actual part of the visualized scenes ("sense of presence": 1 , not at all. . . 5, felt like I was really there).

Scanning parameters. T2*-weighted echo planar images (EPIs) with blood oxygen leveldependent (BOLD) contrast were acquired on a 1.5 tesla Siemens AG (Erlangen, Germany) Sonata MRI scanner. We used standard scanning parameters to achieve whole-brain coverage: 45 oblique axial slices angled at 30 degrees in the anteroposterior axis, $2 \mathrm{~mm}$ thickness $(1 \mathrm{~mm}$ gap), repetition time of $4.05 \mathrm{~s}$. The first six “dummy" volumes from each session were discarded to allow for T1 equilibration effects. A T1weighted structural MRI scan was acquired for each subject after the four main scanning sessions. Images were analyzed in a standard manner using the statistical parametric mapping software SPM5 (www.fil.ion. ucl.ac.uk/spm). Spatial preprocessing consisted of realignment and normalization to a standard EPI template in Montreal Neurological Institute (MNI) space with a resampled voxel size of $3 \times 3 \times 3 \mathrm{~mm}$, and smoothing using a Gaussian kernel with full-width at half-maximum of $8 \mathrm{~mm}$.

Data analysis. After preprocessing, statistical analysis was performed using the general linear model. The experiment had three main "scene" conditions and three baseline "object" conditions. Our interest was in the $16 \mathrm{~s}$ visualization period when subjects were vividly recalling or imagining the scenes or objects. This period was modeled as a boxcar function (of 16 s duration) and convolved with the canonical hemodynamic response function to create regressors of interest. Subject-specific movement parameters were included as regressors of no interest. Subjectspecific parameter estimates pertaining to each regressor (betas) were calculated for each voxel. These parameter estimates (collapsed across sessions) were entered into a second level random-effects analysis using a one-way ANOVA. We report results in a priori regions of interest (previously identified in numerous neuroimaging studies of episodic memory (Maguire, 2001; Rugg et al., 2002; Svoboda et al., 2006; Cabeza and St Jacques, 2007) at $p<0.001$, uncorrected for multiple comparisons, with an extent threshold of 5 or more contiguous voxels. Activations in other brain regions are reported for completeness at a threshold of $p<0.001$ uncorrected for multiple comparisons, but were only considered significant if they survive whole brain correction for multiple comparisons at $p<0.05$ [in line with established procedures (Frackowiak et al., 2004)] A standard conjunction analysis (Friston et al., 2005), as implemented in SPM5 and reported at $p<0.001$, was performed to reveal the regions activated in common between the main scene conditions compared with their respective individual baseline object conditions. Two additional analyses were also performed in which effects attributable to difficulty, vividness, coherence, and memory were either factored out by including these ratings as covariates of no interest in a second level ANOVA, or treated as covariates of interest. All activations are displayed on sections of the average structural image of all the participants. Reported voxels conform to MNI coordinate space. Right side of the brain is displayed on the right side. 


\section{Results}

\section{Behavioral data}

Ratings summary

During scanning, after each visualization period (Fig. 1) subjects were immediately asked to rate on five-point scales the just (re)constructed scene or object across four measures (see Materials and Methods). Overall the conditions were generally very well matched (Table 2). All conditions were rated as low in difficulty (all conditions had a mean $<1.9$ ) and the images visualized as vivid (all conditions had a mean $>3.9$ ). The scenes (re)created as part of the three main conditions (RM, IS, NS) were all rated to be coherent and contiguous (all had a mean >3.9), conversely, the background contexts in the three object control conditions ( $\mathrm{RO}$, $\mathrm{IO}, \mathrm{NO}$ ) were rated as fragmentary (all object conditions had a mean coherence $<1.8$ ), indicating that instructions to minimize context and focus solely on visualizing the required object had been adhered to. Finally, the four "memory" conditions (both real and previously imagined: RM, RO, IS, IO) that required recall of a memory elicited/established in the previous interview session were well remembered (all had a mean $>3.6$ ). The two "new" conditions (NS, NO) did not rely heavily on explicit episodic memories (each had a mean memory rating $<2.0$ ), thus confirming that instructions not to use a memory or any part of one or something they planned to do but instead to create something new had been followed.

\section{Formal comparisons}

As expected, all three main scene conditions were rated as more coherent than their respective object baselines $\left(\mathrm{RM}>\mathrm{RO}, t_{(20)}=\right.$ $13.52, p<0.001$; IS $>$ IO, $t_{(20)}=13.60, p<0.001$; NS $>$ NO, $t_{(20)}$ $=11.03, p<0.001)$ thus confirming that objects had been visualized acontextually as instructed. RM was rated as easier, more vivid and more like a real memory than the pictures of objects seen in the interview session $(\mathrm{RO})\left(\mathrm{RM}>\mathrm{RO}\right.$; difficulty: $t_{(20)}=$ $-4.89, p<0.001$; vividness: $t_{(20)}=4.36, p<0.001$; memory: $t_{(20)}$ $=4.41, p<0.001)$. Also, imagining new scenes (NS) was rated as significantly harder than imagining new objects (NO) $\left(t_{(20)}=\right.$ $2.88, p<0.01)$. There were no other significant differences.

Direct comparison of the three main scene conditions with each other revealed no significant difference in difficulty, vividness or coherence between any of the conditions except for recalling real autobiographical memories (RM) which was rated as significantly easier and more vivid than the other two scene conditions (difficulty: RM $>$ IS, $t_{(20)}=-4.77, p<0.001, \mathrm{RM}>\mathrm{NS}$, $t_{(20)}=-6.67, p<0.001$; vividness: $\mathrm{RM}>\mathrm{IS}, t_{(20)}=5.51, p<$ $\left.0.001, \mathrm{RM}>\mathrm{NS}, t_{(20)}=4.96, p<0.001\right)$. Real memories were also better remembered (i.e., had a higher memory rating) than imaginary memories (IS) $\left(t_{(20)}=6.17, p<0.001\right)$. Of course new imagined experiences (NS) relied on actual memories significantly less than the recall of real (RM) or imaginary memories (IS) $\left(\mathrm{RM}>\mathrm{NS}, t_{(20)}=17.85, p<0.001\right.$; IS $>\mathrm{NS}, t_{(20)}=11.18$, $p<0.001)$ thus confirming that subjects had adhered to instructions to create something completely new and fictitious for the NS condition.

Direct comparison of the three object control conditions (RO, $\mathrm{IO}, \mathrm{NO})$ with each other revealed no significant difference in difficulty, vividness, coherence or memory except for recalling previously imagined objects $(\mathrm{IO})$ which was rated as harder than imagining new objects (NO) $\left(t_{(20)}=2.42, p<0.03\right)$. Again, as with the scenes, new imagined objects (NO) relied on actual memories significantly less than the recall of real ( $\mathrm{RO})$ or imaginary objects $(\mathrm{IO})\left(\mathrm{RO}>\mathrm{NO}, t_{(20)}=12.99, p<0.001\right.$; $\mathrm{IO}>\mathrm{NO}$, $\left.t_{(20)}=8.67, p<0.001\right)$ thus confirming that subjects had adhered to instructions to create new fictitious objects for the NO condition.

The pattern of performance observed with the real memory (RM) condition was expected, as retrieving a recent real salient personal memory and vividly reliving it is not a difficult task for a healthy subject. It should be noted that although RM condition was rated as easier, more vivid and better remembered, this was in the context of all other tasks being rated as easy, salient and well remembered also.

At the interview session 1 week before scanning, subjects were asked to rate the vividness and the use of memory after each imagined scene they constructed. There was no significant difference in vividness between the scenes constructed in the interview (mean $\pm \mathrm{SD}, 3.98 \pm 0.46$ ) when compared with those same scenes later recalled in the scanner (IS) $\left(t_{(20)}=-0.89, p=0.39\right.$ ) nor compared with new scenes freshly created in the scanner for the first time (NS) $\left(t_{(20)}=0.51, p=0.62\right)$. There was also no significant difference between the (low) use of episodic memory in imagining a new scene for the first time whether done in the interview session (mean $\pm \mathrm{SD}, 1.75 \pm 0.31$ ) or in the scanner $($ mean $\pm \mathrm{SD}, 1.94 \pm 0.33)\left(t_{(20)}=-1.97, p=0.06\right)$ again confirming that in both cases something new had been created.

Overly positive or negative real autobiographical memories were discarded at the interview stage, as were memories that the subject could not visualize saliently or pinpoint accurately in time. All real memories taken forward to scanning were therefore recent (mean $\pm \mathrm{SD}, 12.82 \pm 2.77 \mathrm{~d}$ old), emotionally neutral, specific in time and vividly recollected. For the imagination conditions scenes and objects were counter-balanced across subjects for those that were presented during the prescan interview (IS, IO) and those that were presented for the first time in the scanner (NS, NO).

\section{Debriefing}

After scanning, subjects were thoroughly debriefed.

Scenes. Subjects were asked how emotional the three main scene conditions made them feel overall on a scale from -3 (negative) to +3 (positive). As expected, the subjects rated all 3 conditions as being emotionally neutral (RM, mean $\pm \mathrm{SD}, 0.24 \pm$ 1.22 ; IS, mean $\pm \mathrm{SD}, 0.62 \pm 0.86$; NS, mean $\pm \mathrm{SD}, 0.38 \pm 0.67)$, with no significant difference between any of the conditions $\left(\mathrm{RM}>\mathrm{IS}, t_{(20)}=-1.00, p=0.33 ; \mathrm{RM}>\mathrm{NS}, t_{(20)}=-0.47, p=\right.$ 0.64 , IS $>$ NS, $\left.t_{(20)}=1.75, p=0.10\right)$.

Subjects were also asked to rate (scale of $1-5$, in which 1 is low) how much they felt themselves to be an actual part of the visualized scenes ("sense of presence"). As expected, real memories were associated with a stronger sense of presence than imagined scenes whether recalled or newly created in the scanner (RM, mean $\pm \mathrm{SD}, 4.32 \pm 0.66$; IS, mean $\pm \mathrm{SD}, 3.81 \pm 0.73$; NS, 
Table 3. Recall of real episodic memories $>$ recall of previously seen objects (RM > RO)

\begin{tabular}{lll}
\hline Region & Peak coordinate $(x, y, z)$ & $Z$ \\
\hline Medial prefrontal cortex & $-3,51,24$ & 5.00 \\
Ventromedial prefrontal cortex & $3,42,-9$ & 4.79 \\
Right superior frontal sulcus & $27,36,39$ & 4.39 \\
Left middle temporal cortex & $-57,-9,-21$ & 4.10 \\
Right middle temporal cortex & $57,-6,-24$ & 3.96 \\
Left hippocampus & $-18,-21,-15$ & 4.54 \\
Right hippocampus & $18,-24,-12$ & 4.19 \\
Right thalamus & $15,-18,9$ & 4.30 \\
Left parahippocampal gyrus & $-18,-36,-15$ & 4.28 \\
Right parahippocampal gyrus & $24,-33,-18$ & 3.97 \\
Left retrosplenial cortex & $-3,-57,15$ & 6.04 \\
Right retrosplenial cortex & $12,-60,12$ & 6.11 \\
Posterior cingulate cortex & $-3,-54,33$ & 6.39 \\
Precuneus & $3,-66,39$ & 5.67 \\
Left posterior parietal cortex & $-48,-78,18$ & 4.46 \\
Right posterior parietal cortex & $48,-72,27$ & 5.71 \\
Medial posterior parietal cortex & $3,-63,63$ & 4.94 \\
\hline
\end{tabular}

mean $\pm \mathrm{SD}, 3.62 \pm 0.79 ; \mathrm{RM}>\mathrm{IS}, t_{(20)}=3.86, p<0.001 ; \mathrm{RM}>$ NS, $t_{(20)}=4.26, p<0.001$; IS $>$ NS, $\left.t_{(20)}=1.09, p=0.29\right)$.

The scenes visualized in the three main conditions were well matched for number of details, with no significant difference between any of the conditions (RM, mean $\pm \mathrm{SD}, 7.12 \pm 1.51)$; IS, mean $\pm \mathrm{SD}, 7.36 \pm 1.85$; NS, mean $\pm \mathrm{SD}, 7.29 \pm 1.59$; RM $>\mathrm{IS}$, $t_{(20)}=-0.69, p=0.50 ; \mathrm{RM}>\mathrm{NS}, t_{(20)}=-0.59, p=0.56$; IS $>$ NS, $\left.t_{(20)}=0.26, p=0.80\right)$. Also, when describing details of the previously imagined scenes (IS), during debriefing, subjects mentioned an average of $89.7 \%$ of the same details they had produced when creating the scene originally in the prescan interview, thus indicating that they had recalled the previously imagined scenes (IS) with a high degree of accuracy and thereby confirming the behavioral rating (memory rating, mean = 3.97(0.68)) obtained in the scanner.

Objects. Memory for both the seen (RO) and imagined objects (IO) from the prescan interview was tested. All objects in the study had a number of salient features [e.g., color, texture, adornments (see Materials and Methods)]. When cued in the scanner only the basic name of the object was given. In the debriefing session an object was regarded as accurately remembered if the subject was able to name correctly and unprompted at least two salient features of the object. Objects were well remembered with a mean $\pm \mathrm{SD}$ of $8.33 \pm 1.35$ and $8.43 \pm 1.29$ of the seen and imagined objects remembered respectively of a possible 10 , and no significant difference between the two conditions $\left(t_{(20)}=\right.$ $-0.30, p=0.77)$.

\section{Neuroimaging data}

\section{Overall brain networks}

To appreciate the overall network associated with each scene condition, we first contrasted each with its respective control condition. By contrasting the recall of recent episodic memories to the recall of acontextual objects previously seen in the interview session $(\mathrm{RM}>\mathrm{RO})$ we replicated the well established episodic memory retrieval network consistently activated in studies of autobiographical memory (Maguire, 2001; Rugg et al., 2002; Svoboda et al., 2006; Cabeza and St Jacques, 2007) (Table 3, Fig. 2A), consisting of the hippocampus bilaterally, parahippocampal gyrus (PHG), retrosplenial cortex, precuneus, posterior cingulate cortex (PCC), posterior parietal cortex, right thalamus, middle temporal cortex bilaterally, and medial prefrontal cortex.

By contrasting the construction of imagined scenes to the imagination of acontextual objects, both newly imagined in the scanner $(\mathrm{NS}>\mathrm{NO}$ ), we identified brain regions involved in the process of creating and imagining vivid fictitious scenes (Table 4, Fig. $2 B$ ) including the right hippocampus, PHG, retrosplenial and posterior parietal cortices. The involvement of the hippocampus provides additional evidence that the process of scene construction involves the hippocampus, consistent with findings in Hassabis et al.'s recent neuropsychological study (Hassabis et al., 2007).

Contrasting the recall of imagined scenes to the recall of imagined acontextual objects, both previously constructed in the interview session before scanning (IS > IO), revealed a similar network as that engaged during the creation of new constructed scenes (i.e., NS > NO) (Table 5, Fig. 2C), but with additional activations in the medial superior frontal gyrus (BA9), and also precuneus and PCC.

Although not the main focus, for completeness we report that the brain regions showing increased activity for objects compared with scenes $(\mathrm{RO}+\mathrm{IO}+\mathrm{NO}>\mathrm{RM}+\mathrm{IS}+\mathrm{NS})$ (supplemental Fig. S1, available at www.jneurosci.org as supplemental material) included lateral occipital cortex (LOC) bilaterally $(-45,-66,-$ $9 ; \mathrm{z}=5.28 ; 42,-69,-3 ; \mathrm{z}=4.90)$, intraparietal sulcus (IPS) bilaterally $(-54,-30,42 ; \mathrm{z}=5.24 ; 51,-33,45 ; \mathrm{z}=4.89)$, and right lateral prefrontal cortex $(39,48,15 ; z=3.96)$. This is strikingly consistent with other studies that have investigated objects compared with places (Sugiura et al., 2005), and has been attributed to greater attention and focus being directed toward the object when present in isolation (Sugiura et al., 2005).

\section{Commonalities: the core scene construction network}

Having observed in the separate contrasts above similarities in the brain networks underpinning the three scene conditions, we next sought to formally examine commonalities between them. A conjunction analysis (Friston et al., 2005) of the main conditions (RM, IS, NS) against their respective controls (RO, IO, NO) allowed us to isolate the common brain network involved in the underlying core process of constructing, maintaining and visualizing complex scenes. This network (Table 6, Fig. 3) included bilateral hippocampi, parahippocampal gyrus, retrosplenial cortex, precuneus, posterior parietal cortex and ventromedial prefrontal cortex (vmPFC).

\section{Differences: additional requirements for real episodic memories}

Having established the brain areas the scene conditions share in common, we next examined differences between them. Specifically, we asked which brain regions distinguished between the recall of real and fictitious events. This was addressed by contrasting the recall of real memories with the recall of previously imagined memories [minus their respective baseline conditions to control for any effects of external versus internally generated stimuli and creative processes: $(\mathrm{RM}-\mathrm{RO})>(\mathrm{IS}-\mathrm{IO})]$. The precuneus, PCC and anterior medial prefrontal (amPFC; BA 10) were preferentially engaged for real compared with imagined memories (Table 7, Fig. 4A). The plots of the parameter estimates in these three areas (Fig. $4 B-D$ ) clearly show that activation in these areas is most sensitive to the recall of real memories. No changes in activity were observed in the reverse contrast [(IS IO) $>(\mathrm{RM}-\mathrm{RO})]$.

We were also able to ask another question, namely which brain areas were differentially engaged by the retrieval compared 
A
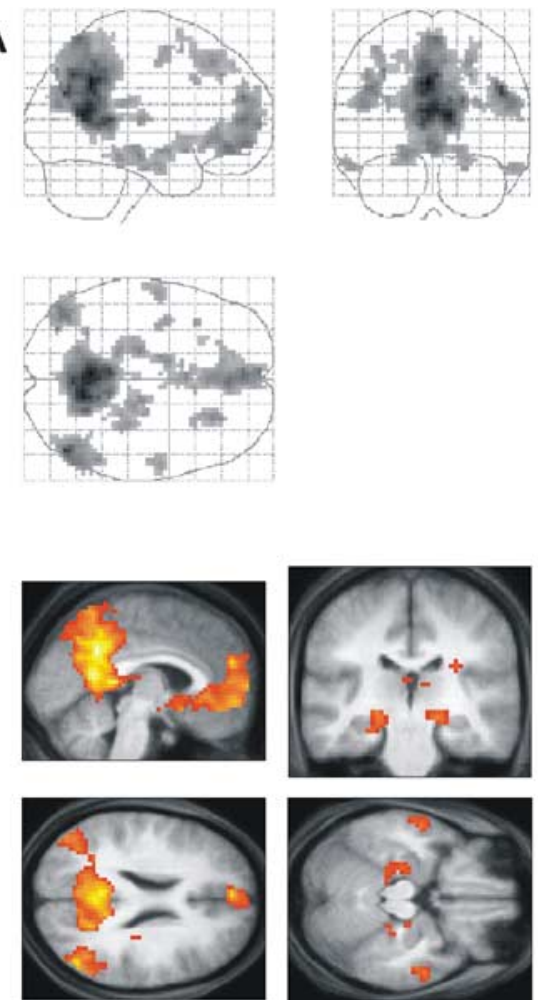

B
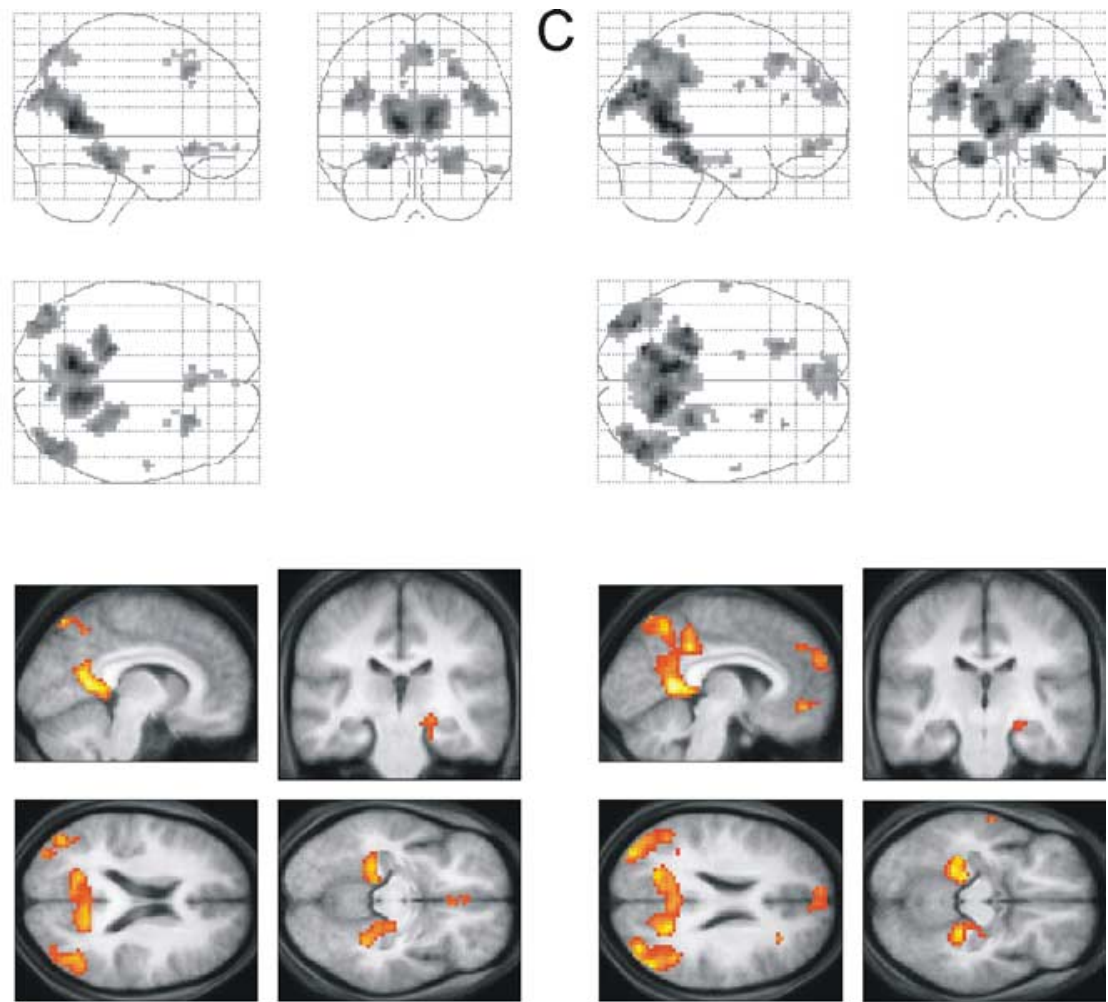

Figure 2. Comparison of the main scene conditions with their respective object baselines. The top row shows sagittal, coronal, and axial images from a "glass brain," which enables one to appreciate activations in all locations and levels in the brain simultaneously. The bottom row shows activations on a selection of relevant sagittal, coronal, and axial sections from the averaged structural MRI scan of the 21 subjects, at a threshold of $p<0.001$ uncorrected. $A, R M>R O$, This contrast reveals the well established network for episodic memory retrieval that includes bilateral hippocampi, parahippocampal gyrus, retrosplenial and posterior parietal cortices, right thalamus, middle temporal cortices, and medial prefrontal cortex. Table 3 details the coordinates of all the activation peaks. $\boldsymbol{B}, \mathrm{NS}>\mathrm{N} 0$, This contrast reveals a similar network for imagining new fictitious experiences that includes right hippocampus, parahippocampal gyrus, retrosplenial and posterior parietal cortices, and ventromedial prefrontal cortex. Table 4 details the coordinates of all the activation peaks. $C, \mid S>10$, This contrast also reveals a similar network for recalling imagined fictitious experiences previously created in a prescan interview that includes right hippocampus, parahippocampal gyrus, retrosplenial and posterior parietal cortices, and medial prefrontal cortex. Table 5 details the coordinates of all the activation peaks.

Table 4. Newly imagined fictitious experiences $>$ newly imagined objects (NS > NO)

\begin{tabular}{lll}
\hline Region & Peak coordinate $(x, y, z)$ & $Z$ \\
\hline Ventromedial prefrontal cortex & $3,24,-9$ & 4.27 \\
Right superior frontal sulcus & $27,27,45$ & 4.42 \\
Right middle temporal cortex & $57,-6,-24$ & 3.70 \\
Right hippocampus & $21,-24,-12$ & 3.86 \\
Left parahippocampal gyrus & $-18,-36,-15$ & 4.28 \\
Right parahippocampal gyrus & $33,-42,-12$ & 4.43 \\
Left retrosplenial cortex & $-12,-60,9$ & 6.08 \\
Right retrosplenial cortex & $12,-57,15$ & 5.52 \\
Right precuneus & $9,-57,48$ & 3.91 \\
Left posterior parietal cortex & $-48,-78,24$ & 4.75 \\
Right posterior parietal cortex & $45,-66,24$ & 4.75 \\
Medial posterior parietal cortex & $9,-75,57$ & 4.73 \\
\hline
\end{tabular}

with the encoding, of an (fictitious) episodic-like event. We contrasted the recall of previously imagined memories to the encoding of newly imagined events (IS $>$ NS) which revealed increased activity in the precuneus bilaterally $(-12,-63,30 ; \mathrm{z}=4.21 ; 9,-$ $69,36 ; z=3.95)$ for recall of previously imagined scenes. This effect is also apparent in the parameter estimates plot in Figure $4 D$. No changes in activity were observed in the reverse contrast (NS $>$ IS).

Additional analyses

Although the ratings for the conditions were overall quite similar in terms of difficulty, vividness, coherence, and memory, we nev-
Table 5. Recall of previously imagined experiences $>$ recall of previously imagined objects (IS > 10)

\begin{tabular}{lll}
\hline Region & Peak coordinate $(x, y, z)$ & $Z$ \\
\hline Medial superior frontal gyrus & $-3,45,36$ & 4.53 \\
Ventromedial prefrontal cortex & $-6,48,-9$ & 4.35 \\
Left superior frontal sulcus & $-24,24,51$ & 4.48 \\
Left middle temporal cortex & $-63,-12,-18$ & 3.93 \\
Right hippocampus & $24,-21,-15$ & 3.49 \\
Left parahippocampal gyrus & $-21,-39,-18$ & 5.57 \\
Right parahippocampal gyrus & $27,-36,-18$ & 5.17 \\
Left retrosplenial cortex & $-9,-48,6$ & 5.87 \\
Right retrosplenial cortex & $15,-57,9$ & 6.21 \\
Left posterior cingulate cortex & $-9,-42,36$ & 5.29 \\
Precuneus & $-3,-60,54$ & 4.88 \\
Left posterior parietal cortex & $-36,-75,30$ & 5.72 \\
Right posterior parietal cortex & $45,-69,33$ & 5.51 \\
Medial posterior parietal cortex & $3,-66,60$ & 4.71 \\
\hline
\end{tabular}

ertheless included the ratings as covariates of no interest in a second level ANOVA in a separate analysis (see Materials and Methods). However, this made no qualitative difference to the results, which was expected, because of the high degree of similarity between the ratings across conditions. In another analysis we treated the ratings as covariates of interest, asking whether activity in any brain areas correlated with the ratings. There were no significant findings, again most likely because the tasks were generally well matched. 
Table 6. Conjunction analysis

\begin{tabular}{llc}
\hline Region & Peak coordinate $(x, y, z)$ & $Z$ \\
\hline Medial prefrontal cortex & $-6,54,27$ & 4.52 \\
Ventromedial prefrontal cortex & $-3,48,-12$ & 3.90 \\
Left superior frontal sulcus & $-24,24,51$ & 5.37 \\
Right superior frontal sulcus & $24,24,48$ & 5.54 \\
Left middle temporal cortex & $-63,-9,-18$ & 4.29 \\
Right middle temporal cortex & $57,-3,-24$ & 4.67 \\
Left hippocampus & $-21,-21,-21$ & 3.90 \\
Right hippocampus & $24,-21,-15$ & 5.04 \\
Left parahippocampal gyrus & $-18,-33,-15$ & 7.52 \\
Right parahippocampal gyrus & $21,-33,-18$ & 5.51 \\
Left retrosplenial cortex & $-6,-54,9$ & $>8$ \\
Right retrosplenial cortex & $12,-54,9$ & $>8$ \\
Posterior cingulate cortex & $-3,-39,42$ & 5.23 \\
Left precuneus & $-6,-63,51$ & 6.12 \\
Right precuneus & $6,-66,60$ & 5.71 \\
Left posterior parietal cortex & $-33,-78,39$ & 6.60 \\
Right posterior parietal cortex & $48,-72,27$ & $>8$ \\
Medial posterior parietal cortex & $-6,-78,54$ & 4.94 \\
\hline
\end{tabular}

The three contrasts entered into the conjunction analysis were: RM - RO, IS - I0, and NS - NO.

\section{Discussion}

In this fMRI study we reveal the extended brain network, including the hippocampus, associated with scene construction, a key process in common to real and imaginary memories, and crucial to the recollective experience of recalling an episodic memory (Tulving, 2002). Moreover, by using rich imaginary memories as a well matched comparison task we were able to isolate processes specific to episodic memory, such as the use of self-schema (Conway and Pleydell-Pearce, 2000; Gallagher, 2000), a sense of familiarity and mental time travel (Tulving, 2002). Previous studies that have compared episodic memory and future thinking were unable to make this distinction because these features were present to a similar degree in both autobiographical memories and personal future scenarios (Atance and O'Neill, 2001; Addis et al., 2007; Szpunar et al., 2007).Together, therefore, the present study advances efforts to dissociate the functions of the many brain regions consistently activated in studies of episodic memory recall (Maguire, 2001; Svoboda et al., 2006; Cabeza and St Jacques, 2007) and episodic future thinking (Atance and O'Neill, 2001; Addis et al., 2007; Szpunar et al., 2007), an endeavor given all the more importance by recent observations that this distributed network may support not just episodic memory, but also numerous other high-level cognitive functions (Buckner and Carroll, 2007; Hassabis and Maguire, 2007).

Our results show that when subjects construct new fictitious scenes an extended brain network involving the hippocampus, parahippocampal gyrus, retrosplenial cortices, posterior parietal cortices, and ventromedial prefrontal cortex is active. Critically, we show using a conjunction analysis that this network is not specific to the construction of new fictitious experiences, but is also engaged when subjects remember both previously imagined experiences as well as their own real personal experiences. The implication is, therefore, that this distributed network supports cognitive operations engaged in common during the three main conditions in our experiment, most likely the (re)construction, maintenance and visualization of complex scenes. Importantly, these effects cannot be attributed to differences in either difficulty or vividness between the conditions given that these variables were generally comparable. A key aspect of our experimental design was the use of control conditions involving the vivid visualization of acontextual objects which allowed us to distinguish between the processes of "simple" imagery (Kosslyn et al., 2001) (e.g., of objects), and that of complex scene construction and visualization, and attribute the latter to a distributed network including the hippocampus (Hassabis et al., 2007). Indeed, our finding of significantly greater activation in the object conditions (i.e., Objects $>$ Scenes) in brain areas often associated with supporting object representations and manipulations (Sugiura et al., 2005) (i.e., LOC and IPS), lends additional support to our assertion that scene construction represents a dissociable cognitive process with a distinct neural basis. As such, a large portion of the episodic memory recall network (Maguire, 2001; Svoboda et al., 2006) would seem to be more accurately characterized as the network responsible for the construction of complex scenes. Given this, it would seem reasonable to propose that real memories are reconstructed along very similar lines to the way imagined events are constructed, dovetailing with the idea that memory is (re)constructive in nature (Bartlett, 1932; Schacter et al., 1998; Conway and Pleydell-Pearce, 2000; Rubin et al., 2003; Hassabis and Maguire, 2007; Schacter and Addis, 2007).

Our data, therefore, also accord well with a recent neuropsychological study (Hassabis et al., 2007) in which hippocampaldamaged patients were found to be impaired at imagining new fictitious experiences. Their descriptions were fragmented, lacked a coherent spatial context, and were significantly less rich suggesting that the hippocampus plays a critical role in integrating the disparate elements of a scene into a coherent whole (O'Keefe and Nadel, 1978; Burgess et al., 2001; Eichenbaum, 2004; Moscovitch et al., 2005; Hassabis and Maguire, 2007; Hassabis et al., 2007). Moreover, evidence from a previous EEG study (Conway et al., 2003) also suggests that common processes underlie the recall of both real and imaginary events.

Another key aim of this study was the investigation of areas differentially engaged by episodic memories and (re)construction of imagined fictitious experiences. When constructing fictitious experiences subjects were instructed not to describe a memory or something they planned to do but instead create something completely new. That novel and original experiences were constructed is evidenced by the low scores obtained on the "use of memory" behavioral measure. Furthermore, it is unlikely that subjects engaged in mental time travel when describing their fictitious scenes because comparisons of the fictitious scene descriptions obtained in this study (supplemental Table S1, available at www.jneurosci.org as supplemental material) to typical descriptions obtained in future thinking studies (Addis et al., 2007, their Appendix A.2) show clear qualitative differences. In future event descriptions temporal phrases such as "I will be", "I plan to" and "I'm going to", and familiar objects and people (e.g., "my sister is there") are commonplace (Addis et al., 2007) in contrast to imagined fictitious scenes in which generally none of these features are present. We therefore sought to capitalize on the absence of self-projection in time and reduced reliance on self-schema processes in the imagination task to identify brain regions that might support these specific qualities of episodic memory, both past and future (Schacter and Addis, 2007). Contrasting the retrieval of recent real episodic memories to the retrieval of recently constructed fictitious experiences, while controlling for external versus internal generation effects with the respective baselines, revealed increased activity in the amPFC, PCC and precuneus. It should be noted that this differential activity cannot be attributable to differences in retrieval effort, as recalling real memories was rated as easier than the other conditions. It is also unlikely to be explained by vividness; although real memories were rated as more vivid, this is set against an overall 
context of high vividness across all conditions. This was confirmed by our analysis in which covarying out the effect of vividness made no qualitative difference to the regions activated in the contrasts. Interestingly, we also observed significantly greater activation in precuneus when we compared the recall of previously imagined experiences with the creation of new imagined experiences. As such, activation in the precuneus may reflect the relative familiarity of the visualized experience [i.e., RM > IS $>$ NS (Fig. 4D)] with real memories, perhaps not surprisingly given their often highly familiar content, more familiar than imaginary memories, in line with proposals concerning the function of this region derived from studies of recognition memory (Rugg et al., 2002; Wagner et al., 2005; Hornberger et al., 2006; Vincent et al., 2006).

Activation in amPFC and PCC, however, was only observed during episodic memory recall, suggesting that these regions support functions that are specific to episodic memory over and above scene construction (Tulving, 2002). In fact the pattern of activation revealed by this contrast bears a striking resemblance to networks found to support self-reflection (Johnson et al., 2002), theory of mind (Kumaran and Maguire, 2005; Amodio and Frith, 2006) and episodic future thinking (Addis et al., 2007) suggesting that the amPFC and PCC might be supporting pro-

cesses related to the self (Conway and Pleydell-Pearce, 2000) and mental time travel (Wheeler et al., 1997; Tulving, 2002). Indeed, when viewed in terms of component processes, episodic future thinking (Atance and O'Neill, 2001) and recalling past events share many of the same underlying processes. These include not only scene construction (which they have in common with imagining fictitious experiences) but also self-schema processing, selfprojection in time (forwards instead of backwards in the case of future thinking), and a sense of familiarity - processes present in imagined fictitious experiences either to a much lesser extent or not at all. Perhaps it is not altogether surprising therefore that recent fMRI studies have found comprehensive overlap in the activation patterns associated with recalling the past and thinking about plausible self-relevant future events (Addis et al., 2007; Szpunar et al., 2007). Together, then, we suggest that during episodic memory retrieval (and perhaps also episodic future thinking), the interaction or cooperation between the self-processing and familiarity functions performed by the amPFC/PCC and precuneus respectively may be sufficient to distinguish between real and fictitious memories and likely reflects the greater sense of presence reported by our subjects for real memories.

Intriguingly, similar brain networks to the classic episodic memory network (also replicated in this study with the RM $>$ RO contrast) has also been activated in a variety of other circumstances including navigation (Burgess et al., 2002; Hartley et al., 2003; Spiers and Maguire, 2006), spatial tasks (Maguire et al., 2003; Kumaran and Maguire, 2005), episodic future thinking (Atance and O'Neill, 2001; Addis et al., 2007; Schacter and Addis,
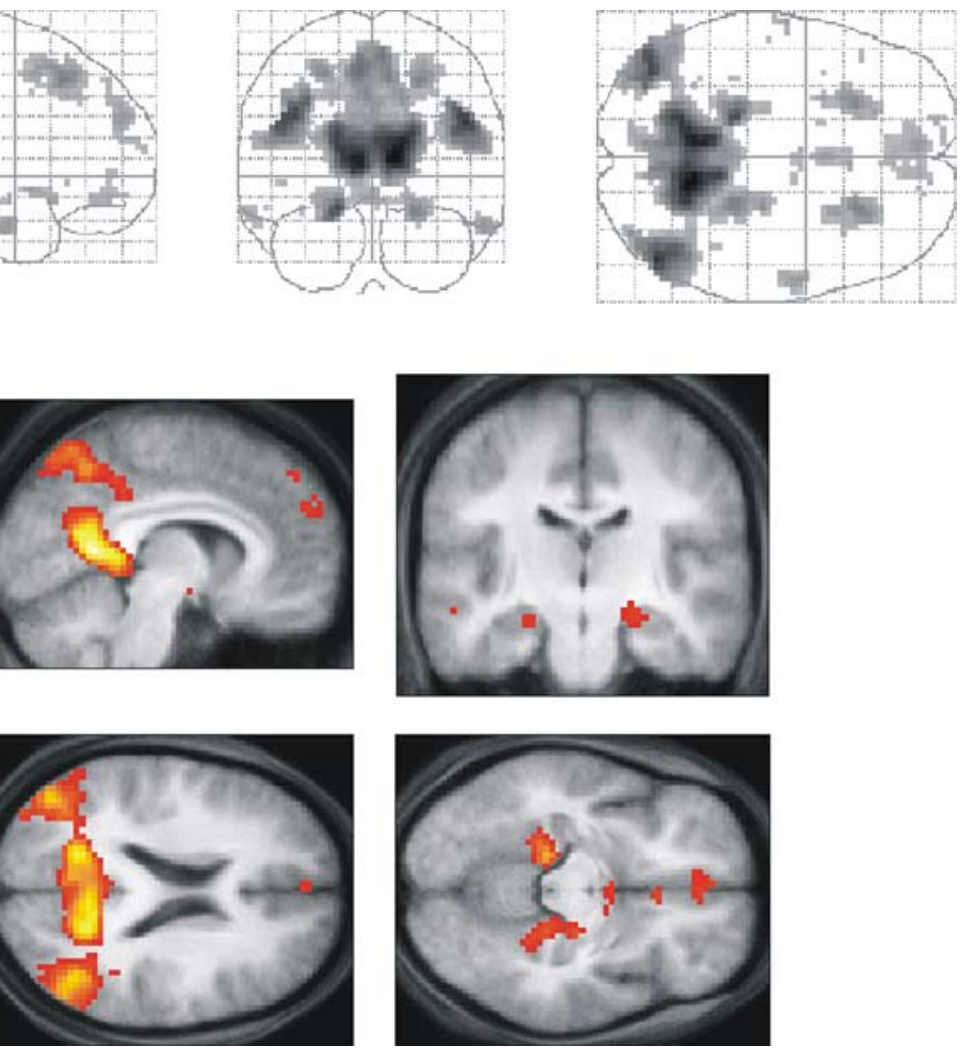

Figure 3. Brain areas in common to the three scene conditions. A conjunction analysis revealed the brain regions activated in peaks. Views of this distributed brain network are also shown in the bottom on a selection of relevant sagittal, coronal, and axial sections from the averaged structural MRI scan of the 21 subjects, at a threshold of $p<0.001$ uncorrected.

Table 7. Recall of real episodic memories $>$ recall of previously imagined experiences $[(\mathrm{RM}-\mathrm{RO})>(\mathrm{IS}-\mathrm{IO})]$

\begin{tabular}{lll}
\hline Region & Peak coordinate $(x, y, z)$ & $Z$ \\
\hline Anterior medial prefrontal cortex & $-3,63,6$ & 4.54 \\
Posterior cingulate cortex & $-3,-54,36$ & 4.61 \\
Precuneus & $3,-63,39$ & 5.21 \\
\hline
\end{tabular}

2007; Szpunar et al., 2007), the default mode (Raichle et al., 2001) and daydreaming (Mason et al., 2007), leading to suggestions that it performs a general function (Buckner and Carroll, 2007; Hassabis and Maguire, 2007). One hypothesis that has recently been proposed relates the function of this brain network to the process of "self projection" (Buckner and Carroll, 2007), a wide-ranging term that encompasses numerous processes including selfrelevance, self-schema or narrative self, scene construction, mental simulation and projection of the self into space, time or onto another's perspective (Tulving, 2002; Buckner and Carroll, 2007). The current findings suggest that this multifaceted concept can be broken down into at least two distinct components with dissociable neural bases: a network centered on the hippocampus responsible for scene construction, with the amPFC/ PCC and precuneus mediating self projection in time, sense of familiarity and self-schema. The disparate cognitive functions highlighted by Buckner and Carroll (2007) and Hassabis and Maguire (2007) may be differentially reliant on these two components (e.g., navigation likely predominantly relies on the scene construction process, whereas episodic memory requires both). 

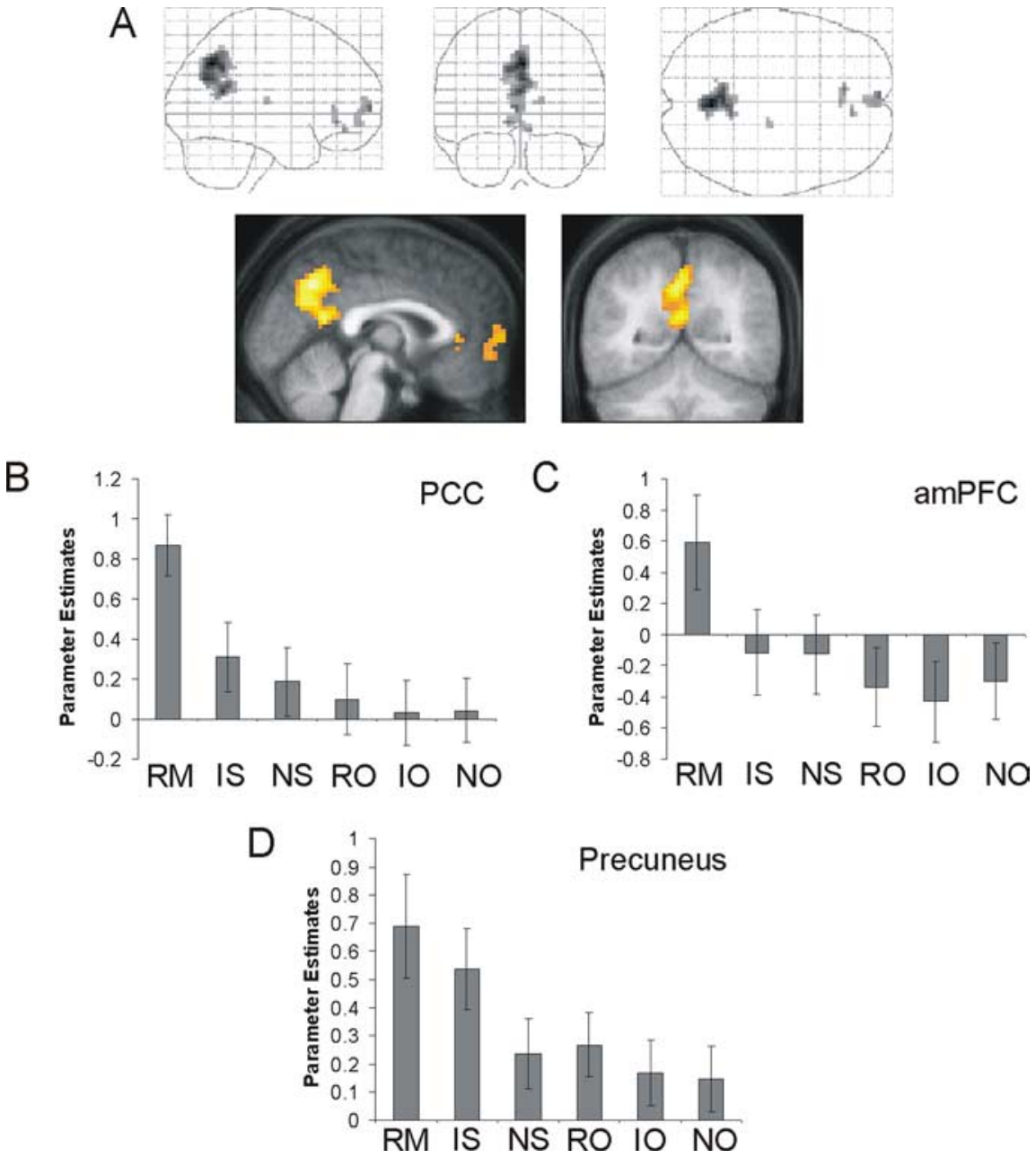

Figure 4. Comparison of real and imagined memories. $A$, Contrasting recall of real memories to the recall of imaginary memories while controlling for externally versus internally generated stimuli and creativity processes, (RM $-\mathrm{RO})>(I S-10)$, revealed that the precuneus, $\mathrm{PCC}$, and amPFC were preferentially engaged for real memories. Table 7 details the coordinates of all the activation peaks. Bottom, Views of these brain regions on a sagittal and coronal section from the averaged structural MRI scan of the 21 subjects, at a threshold of $p<0.001$ uncorrected. $\boldsymbol{B}-\boldsymbol{D}$, Condition-specific parameter estimates (betas) in arbitrary units at the peak voxels. Error bars represent the SE. RM, Recall of real memories; IS, recall of previously imagined scenes; NS, constructing new fictitious scenes; $\mathrm{R} 0$, recall of previously seen objects; 10 , recall of previously imagined objects; $\mathrm{NO}$, imagining new objects. $\boldsymbol{B}$, At the peak voxel in PCC. $\boldsymbol{C}$, At the peak voxel in amPFC. $\boldsymbol{D}$, At the peak voxel in the precuneus.

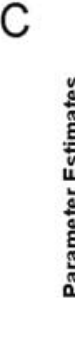

riences is a manipulable and useful experimental tool which may prove fruitful in further advancing our understanding of episodic memory and the processes underpinning it, as well as other related cognitive functions (Buckner and Carroll, 2007; Hassabis and Maguire, 2007). In the future, it will be important to define the exact function of individual regions within the scene construction network, explain the role of these functions in apparently unrelated tasks such as word-pair recognition tasks that also often involve this network, and discover the precise mechanisms which give rise to the phenomenological feeling one experiences during episodic memory recall that allows us to know that an event really happened.

\section{References}

Addis DR, Wong AT, Schacter DL (2007) Remembering the past and imagining the future: common and distinct neural substrates during event construction and elaboration. Neuropsychologia 45:1363-1377.

Amodio DM, Frith CD (2006) Meeting of minds: the medial frontal cortex and social cognition. Nat Rev Neurosci 7:268-277.

Atance CM, O’Neill DK (2001) Episodic future thinking. Trends Cogn Sci 5:533-539.

Bartlett FC (1932) Remembering. Cambridge: Cambridge UP.

Buckner RL, Carroll DC (2007) Self-projection and the brain. Trends Cogn Sci 11:49-57.

Burgess N (2006) Spatial memory: how egocentric and allocentric combine. Trends Cogn Sci 10:551-557.

Burgess N, Becker S, King JA, O'Keefe J (2001) Memory for events and their spatial context: models and experiments. Philos Trans R Soc Lond B Biol Sci 356:1493-1503.

Burgess N, Maguire EA, O’Keefe J (2002) The human hippocampus and spatial and episodic memory. Neuron 35:625-641.

Cabeza R, St Jacques P (2007) Functional neuroimaging of autobiographical memory. Trends Cogn Sci 11:219-227.

To conclude, we have demonstrated that a distributed brain network, including the hippocampus, is recruited during both episodic memory recall and the visualization of fictitious experiences. Our data provide evidence for the role of this brain network in scene construction, a critical process underpinning rich recollection. We further propose that the recruitment of this network in a variety of different conditions (Buckner and Carroll, 2007; Hassabis and Maguire, 2007), ranging from the default state, spatial navigation to episodic future thinking, may in fact reflect its general role in the process of scene construction. Our findings also provide insights into the neural basis of processes specific to episodic memory, suggesting a model in which activation in amPFC, PCC and precuneus interact to allow real memories to be distinguished from imaginary ones. Moreover, the notion that scene construction is a key component of episodic memory recall lends additional support to constructive theories of episodic memory (Bartlett, 1932; Schacter et al., 1998) and the recently proposed constructive episodic simulation function of the episodic memory system (Schacter and Addis, 2007; Schacter et al., 2007). This study demonstrates that imagining new expe-
Conway MA, Pleydell-Pearce CW (2000) The construction of autobio-

Conway MA, Pleydell-Pearce CW, Whitecross SE, Sharpe H (2003) Neurophysiological correlates of memory for experienced and imagined events. Neuropsychologia 41:334-340.

Eichenbaum H (2004) Hippocampus: cognitive processes and neural representations that underlie declarative memory. Neuron 44:109-120.

Frackowiak RSJ, Friston KJ, Frith CD, Dolan RJ, Price CJ, Zeki S, Ashburner JT, Penny WD (2004) Human brain function. New York: Elsevier Academic.

Friston KJ, Penny WD, Glaser DE (2005) Conjunction revisited. NeuroImage 25:661-667.

Frith U, Frith CD (2003) Development and neurophysiology of mentalizing. Philos Trans R Soc Lond B Biol Sci 358:459-473.

Gallagher S (2000) Philosophical conceptions of the self: implications for cognitive science. Trends Cogn Sci 4:14-21.

Gottfried JA, Smith AP, Rugg MD, Dolan RJ (2004) Remembrance of odors past: human olfactory cortex in cross-modal recognition memory. Neuron 42:687-695.

Greenberg DL, Rubin DC (2003) The neuropsychology of autobiographical memory. Cortex 39:687-728.

Hartley T, Maguire EA, Spiers HJ, Burgess N (2003) The well-worn route graphical memories in the self-memory system. Psychol Rev 107:261-288. 
and the path less traveled: distinct neural bases of route following and wayfinding in humans. Neuron 37:877-888.

Hassabis D, Maguire EA (2007) Deconstructing episodic memory with construction. Trends Cogn Sci 11:299-306.

Hassabis D, Kumaran D, Vann SD, Maguire EA (2007) Patients with hippocampal amnesia cannot imagine new experiences. Proc Natl Acad Sci USA 104:1726-1731.

Hornberger M, Rugg MD, Henson RN (2006) fMRI correlates of retrieval orientation. Neuropsychologia 44:1425-1436.

Johnson SC, Baxter LC, Wilder LS, Pipe JG, Heiserman JE, Prigatano GP (2002) Neural correlates of self-reflection. Brain 125:1808-1814.

Kosslyn SM, Ganis G, Thompson WL (2001) Neural foundations of imagery. Nat Rev Neurosci 2:635-642.

Kumaran D, Maguire EA (2005) The human hippocampus: cognitive maps or relational memory? J Neurosci 25:7254-7259.

Maguire EA (2001) Neuroimaging studies of autobiographical event memory. Philos Trans R Soc Lond B Biol Sci 356:1441-1451.

Maguire EA, Valentine ER, Wilding JM, Kapur N (2003) Routes to remembering: the brains behind superior memory. Nat Neurosci 6:90-95.

Mason MF, Norton MI, Van Horn JD, Wegner DM, Grafton ST, Macrae CN (2007) Wandering minds: the default network and stimulusindependent thought. Science 315:393-395.

Moscovitch M, Rosenbaum RS, Gilboa A, Addis DR, Westmacott R, Grady C, McAndrews MP, Levine B, Black S, Winocur G, Nadel L (2005) Functional neuroanatomy of remote episodic, semantic and spatial memory: a unified account based on multiple trace theory. J Anat 207:35-66.

O'Keefe J, Nadel L (1978) The hippocampus as a cognitive map. Oxford: Oxford UP.

Raichle ME, MacLeod AM, Snyder AZ, Powers WJ, Gusnard DA, Shulman GL (2001) A default mode of brain function. Proc Natl Acad Sci USA 98:676-682.

Rosenbaum RS, McKinnon MC, Levine B, Moscovitch M (2004) Visual imagery deficits, impaired strategic retrieval, or memory loss: disentangling the nature of an amnesic person's autobiographical memory deficit. Neuropsychologia 42:1619-1635.
Rubin DC, Schrauf RW, Greenberg DL (2003) Belief and recollection of autobiographical memories. Mem Cognit 31:887-901.

Rugg MD, Otten LJ, Henson RN (2002) The neural basis of episodic memory: evidence from functional neuroimaging. Philos Trans R Soc Lond B Biol Sci 357:1097-1110.

Schacter DL, Addis DR (2007) The cognitive neuroscience of constructive memory: remembering the past and imagining the future. Philos Trans R Soc Lond B Biol Sci 362:773-786.

Schacter DL, Norman KA, Koutstaal W (1998) The cognitive neuroscience of constructive memory. Annu Rev Psychol 49:289-318.

Schacter DL, Addis DR, Buckner RL (2007) Remembering the past to imagine the future: the prospective brain. Nat Rev Neurosci 8:657-661.

Spiers HJ, Maguire EA (2006) Thoughts, behaviour, and brain dynamics during navigation in the real world. NeuroImage 31:1826-1840.

Sugiura M, Shah NJ, Zilles K, Fink GR (2005) Cortical representations of personally familiar objects and places: functional organization of the human posterior cingulate cortex. J Cogn Neurosci 17:183-198.

Svoboda E, McKinnon MC, Levine B (2006) The functional neuroanatomy of autobiographical memory: a meta-analysis. Neuropsychologia 44:2189-2208.

Szpunar KK, Watson JM, McDermott KB (2007) Neural substrates of envisioning the future. Proc Natl Acad Sci USA 104:642-647.

Tulving E (2002) Episodic memory: from mind to brain. Annu Rev Psychol 53:1-25.

Vincent JL, Snyder AZ, Fox MD, Shannon BJ, Andrews JR, Raichle ME, Buckner RL (2006) Coherent spontaneous activity identifies a hippocampal-parietal memory network. J Neurophysiol 96:3517-3531.

Wagner AD, Shannon BJ, Kahn I, Buckner RL (2005) Parietal lobe contributions to episodic memory retrieval. Trends Cogn Sci 9:445-453.

Wheeler MA, Stuss DT, Tulving E (1997) Toward a theory of episodic memory: the frontal lobes and autonoetic consciousness. Psychol Bull 121:331-354.

Wheeler ME, Petersen SE, Buckner RL (2000) Memory's echo: vivid remembering reactivates sensory-specific cortex. Proc Natl Acad Sci USA 97:11125-11129. 